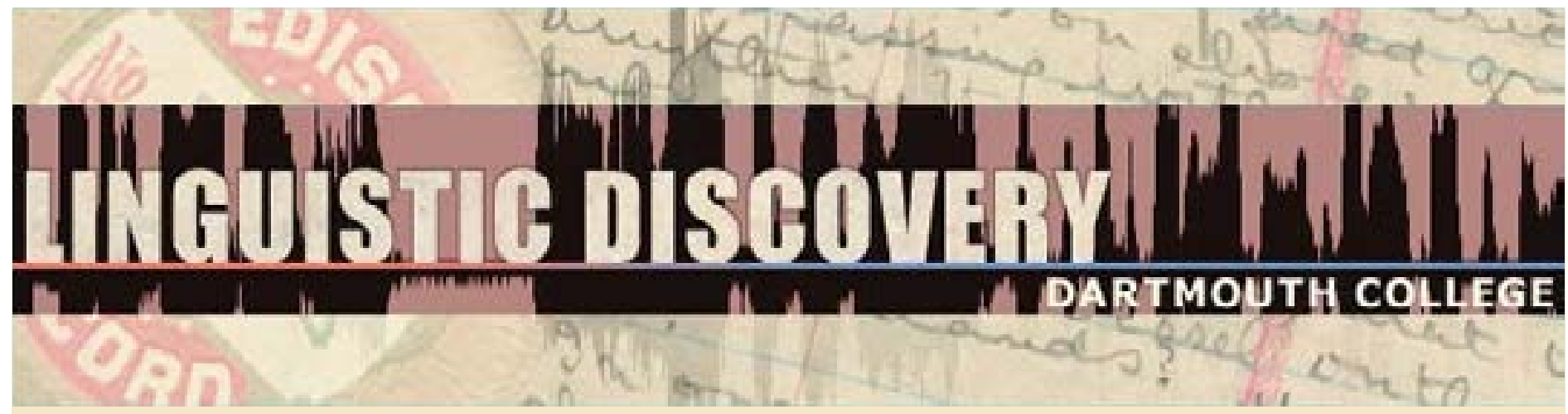

\begin{tabular}{|l|}
\hline Volume 8 \\
Issue 1 \\
2010 \\
\hline
\end{tabular}

\title{
Commentary on Cristofaro - What Do Semantic Maps Tell Us?
}

William Croft

University of New Mexico

doi: $10.1349 / P S 1.1537-0852 . A .362$

url: http://journals.dartmouth.edu/cgi-bin/WebObjects/ Journals.woa/1/xmlpage/1/article/362

\section{Linguistic Discovery}

Published by the Dartmouth College Library Copyright to this article is held by the authors. ISSN 1537-0852 linguistic-discovery.dartmouth.edu 


\title{
What Do Semantic Maps Tell Us?
}

\section{Comment on 'Semantic Maps and Mental Representation' by Sonia Cristofaro (2010)}

\author{
William Croft \\ University of New Mexico
}

Cristofaro (henceforth $\mathrm{C}$ ) challenges the hypothesis advocated by many users of the semantic map model in typology, including myself, that the structure of conceptual space in the semantic map model reflects conceptual similarity relationships between situation types that are part of the mental representations of speakers. $\mathrm{C}$ arrives at two general conclusions about the relationship between the semantic map model and mental representations. First, she argues that the putative similarity relationships between the situation types expressed by linguistic forms which are captured in semantic maps are not actually similarity relationships present in a speaker's mental representation of those situation types. Second, she argues that semantic maps "pertain to the principles that govern the creation of novel constructions at the diachronic level, independently of synchronic grammatical representation in a speaker's mind'. In this commentary, I argue that the semantic map model does in fact reflect conceptual similarity relationships which are part of a speaker's knowledge.

C's second conclusion implies a much sharper division between synchrony and diachrony than actually exists. There is not really a 'diachronic level' independent of speaker's knowledge about their language. Diachronic processes begin with speakers making linguistic choices in the process of verbalizing their experiences in utterances (Croft 2000). These choices cannot be made without a speaker using her knowledge about her language, no matter how fragmentary that may be (as in the case of limited second language speakers). If the semantic map model represents something about diachronic processes, then they represent something about a speaker's mental representation, i.e. her knowledge about her language.

It is of course possible that it is not similarity relationships which are represented. C's first conclusion is that the supposed similarity relationships in the semantic map model are not that. Instead, they are the result of processes that do not involve conceptual similarity. $\mathrm{C}$ gives two examples of such processes. The first is the hypothesis that metonymic processes drive certain semantic changes such as grammaticalization and "generalization" (the examples that $\mathrm{C}$ gives all appear to involve metonymy, and "generalization" in the usual sense of that term actually does not play any role in the process). The second is that processing and/or token frequency determines certain typological universals such as the animacy hierarchy, not conceptual similarity relationships among the elements in an implicational hierarchy.

I will start (again) with C's second argument, regarding implicational hierarchies such as the animacy hierarchy. In Radical Construction Grammar (Croft 2001), I proposed that typological markedness patterns, including certain implicational hierarchies, could be captured by aspects of the structure of the conceptual space. However, I realized that was incorrect by the time I wrote the new edition of Typology and Universals (Croft 2003). In the latter book, I argue that typological markedness patterns were a function of properties of the semantic maps - that is, not the conceptual space itself but the language-specific categories that are mapped onto the (presumably universal) conceptual space. Typological markedness is a consequence of the token frequency of the linguistic categories in question. 
For example, nominative case is less marked with respect to structural coding than accusative case because the token frequency of the category mapped by a nominative form (the combination of A plus $S$ roles in the conceptual space of grammatical relations) is higher than the token frequency of the category mapped by an accusative form ( $\mathrm{P}$ in the conceptual space). The absolutive is also less marked in structural coding than the ergative, and that is because the combination of roles mapped by the absolutive ( $\mathrm{S}$ plus $\mathrm{P}$ ) has a higher token frequency than the roles mapped by the ergative (A). What matters is that $\mathrm{A}+\mathrm{S}$ outnumbers $\mathrm{P}$ in token frequency, and $\mathrm{S}+\mathrm{P}$ outnumbers $\mathrm{A}$; and this is a property of the semantic maps, not of the conceptual space itself. More precisely, it does not have to do with the relations between situation types represented by the links in the conceptual space. It is those links that are intended to reflect similarity relations between the situation types in the conceptual space. It does, of course, have to do with the (combined) token frequencies of the individual situation types in the conceptual space.

More generally, in Typology and Universals I argue that there are typological generalizations which have the effect of constraining semantic maps, over and above the constraints imposed on possible semantic maps (i.e. possible linguistic categories) by the links between situation types in a conceptual space. The constraints imposed on possible linguistic categories by the links between situation types are summarized in the Semantic Map Connectivity Hypothesis: that a possible linguistic category (semantic map) is constrained to be a connected region in the conceptual space. The explanation behind the Semantic Map Connectivity Hypothesis is based on the hypothesis that the links represent similarity relationships: situation types are grouped under a single linguistic category only if they are similar enough to each other in the relevant semantic dimensions. But these are not the only constraints on possible semantic maps. That is, the Semantic Map Connectivity Hypothesis is insufficient to describe possible linguistic categories.

For example, in the animacy hierarchy illustrated in (1), not only is it the case that the Semantic Map Connectivity Hypothesis holds (categories must be connected in the linear conceptual space), but that a linguistic category such as plurality must always include the "highest" function in the space, namely 1 st/2nd person:

$$
\text { 1st/2nd person - 3rd person - human } \mathrm{N} \text { - animate } \mathrm{N} \text { - inanimate } \mathrm{N}
$$

One of Cristofaro's examples also illustrates the need for further constraints and explanations for that constraint. She cites König and Siemund's observation that no language in their survey includes the derived intransitivity function under the same marker as reflexives if that marker is also used for the intensifier function, despite the fact that all three are linked in a grammaticalization path/conceptual space as in (2):

$$
\text { Intensifier — Reflexive — Derived intransitivity indicator }
$$

These further constraints call for explanation, of course. (There is a plausible explanation for König and Siemund's observation, incidentally: the process that extends reflexives to the derived intransitivity function is much slower than the process that replaces old intensifiers - which have acquired a reflexive function - with new intensifiers; this latter process is known to be relatively rapid.) But the fact that there are further constraints on possible linguistic categories/semantic maps does not invalidate the Semantic Map Connectivity Hypothesis or the similarity 
relationships that it embodies. For example, it is true that the functions found at the "upper end" of the animacy hierarchy have a greater token frequency: we talk more about ourselves than others, and more about people than nonhuman entities, and so on. For this reason, plurals are more likely to occur in categories at the "upper end" of the animacy hierarchy (that is, such categories have a greater behavioral potential, thanks to their greater token frequency; see Croft 2003). But the frequency effect does not explain why one does not find a language with plural marking for personal pronouns and nonhuman animate nouns but not for human nouns, even though the personal pronoun + animate noun category would have a higher token frequency than the human noun category. In other words, we still need the animacy conceptual space structure, and the similarity relationships it reflects, in order to explain the linguistic categories we find, even if we need other things as well.

I now turn to the more challenging question of what the semantic map model has to say about the sorts of categories that $\mathrm{C}$ describes which appear to involve metonymy. The first question I would like to raise is whether the semantic map model should be expected to apply to these cases at all.

The semantic map model, and more sophisticated models to capture the same phenomena such as multidimensional scaling (Croft and Poole 2008), presumes that the data in question are similarity data. The assumption behind the applicability of the semantic map model and of multidimensional scaling to typological linguistic data is that crosslinguistic category data do provide evidence of similarity relations. Here, the hypothesis is that if a language encodes two situation types under the same linguistic form, then there is some conceptual similarity between those two situation types in the mind of a speaker. This hypothesis seems quite reasonable for many of the linguistic phenomena for which the semantic map model has been used. For example, the implicational hierarchies such as the animacy hierarchy presuppose that the values in question (i.e. the functions) form a set of paradigmatic alternatives (Croft 2003). Haspelmath's semantic map analysis of indefinite pronouns, and the multidimensional scaling analyses of spatial adpositions by Levinson et al. (2003) and Croft and Poole (2008) also involve paradigmatic alternatives. The same is true of the grammatical relations space in Croft (2001); they are all participant roles. In some cases, two related, paradigmatically-defined conceptual dimensions are crossed: in the parts of speech space in Croft (2001), propositional act functions are crossed with lexical semantic classes, and in the tense-aspect space in Croft and Poole (2008), time reference is crossed with aspect (perfectivity).

One could argue that in the examples which $\mathrm{C}$ presents, the hypothesis that formal similarity implies conceptual similarity simply does not hold. The metonymic shifts lead to jumps from one semantic dimension to another, and there is no paradigmatic relationship between the two, for example between body parts and spatial relations, or between time and contrastiveness (adversative). Hence, one could conclude that the semantic map model should not be applied in those cases. In at least one case that $\mathrm{C}$ discusses, a semantic map model would in fact not reveal the pattern in question. This is the case of the exaptation or hypoanalysis of the imperfective as a subjunctive. As C notes (citing Bybee et al. 1994:230-36 and Haspelmath 1998; see also Croft 2000:127-28), this results when a progressive has taken over main clause contexts; the imperfective is restricted to subordinate clause contexts and is reanalyzed as a subjunctive. But the typological data for a semantic map model would not pick this up: the imperfective acquires its subjunctive function after it has lost its imperfective function to the progressive, so one would not find languages with both functions synchronically. The synchronic mutual exclusivity of meanings is the usual case in exapation/hypoanalysis. 
There are certainly other typological phenomena to which the semantic map model (or multidimensional scaling) should not be applied. For example, word order universals do not imply any similarity relation between different word orders (Croft and Poole 2008:10, fn. 1). And perhaps we should be cautious about assuming that subsuming two functions under one form implies that the functions are always similar. But I doubt that $\mathrm{C}$ is really making that argument. For example, her examples of "generalization" could be analyzed as members of a paradigmatic set, of modality (albeit divided into deontic and epistemic modality). But $\mathrm{C}$ argues (following Traugott and Dasher) that the semantic process leading to the grouping of different functions under a single form are due to metonymy, not similarity: the different uses are associated in a particular context of use, and the meaning in the context is transferred to the form that expressed the other meaning. Typological studies of lexical polysemy (Brown 1979; Evans 1992; Croft et al. 2009) demonstrate that some sort of metonymy is a common motivation for the subsumption of different meanings under a single lexical item. For example, the word for 'moon' very frequently also means 'month': this is a metonymic relationship between a celestial object and a temporal cycle associated with that object's behavior. Also, the word for 'night' very frequently also means 'evening': this is a metonymic relationship between a celestially-defined time period and the beginning phase of that period.

So we are back at the ancient opposition between association and similarity, between metonymy and metaphor. The semantic map model assumes the latter, while C's putative counterexamples are claimed to be examples of the former. I have suggested above that precisely because they are not examples of similarity, then there might be reason to say that the semantic map model does not and should not apply to them. C's putative counterexamples certainly do not invalidate the many cases where similarity is a very plausible explanation for the processes of semantic extension which are presumably behind the typological patterns, just as C's observation that similarity is not enough does not invalidate the role that similarity does play in grammatical patterns. However, I would like to suggest that there is in fact not such a strict dichotomy between association and similarity.

Semantic map models generally use very broadly-defined functions, such as "property predication", "ability modality", or "non-specific indefinite pronoun". These categories are in fact generalizations over large classes of specific occurrences of these meanings in situation types produced in utterances. In some studies, however, the functions are much more finegrained. Dahl's tense-aspect typological study (Dahl 1985), on which the tense-aspect spatial model in Croft and Poole (2008) was based, consists of tense-aspect sentences elicited as translations of sentences in a questionnaire. Levinson et al.'s typological study of spatial adpositions, referred to above, uses sentences elicited in a picture description task. These functions are basically utterances produced in a controlled context. These studies point to the logical conclusion of the process: the conceptual space should consist of points representing actual situation types verbalized by linguistic forms in utterances (Croft, to appear). These instances of language use are the exemplars on which semantic knowledge is constructed by the speaker. The speaker's conceptual space consists of all of the situation types she has experienced, related to each other conceptually in ways that are reflected by the choices of linguistic expressions used to verbalize those experiences.

For example, a conceptual space for participant roles might contain two roles defined in a coarse-grained manner, 'instrumental' and 'comitative'. These roles are certainly linked; a common grammaticalization path leads from the latter to the former. They can also be thought of as paradigmatic alternatives. But in fact, particular examples suggest not only that a more fine- 
grained division of participant roles would be desirable, but experimental evidence suggests that the 'comitative'/'instrumental' roles can be ordered in a one-dimensional conceptual space (Schlesinger 1979:310, cited in Heine et al. 1991:104; I am not ruling out the possibility that a higher-dimensional spatial model might be more appropriate in this case):

(3) a. The pantomimist gave a show with the clown.

b. The engineer built the machine with an assistant.

c. The general captured the hill with a squad of paratroopers.

d. The acrobat performed an act with an elephant.

e. The blind man crossed the street with his dog.

f. The officer caught the smuggler with a police dog.

g. The prisoner won the appeal with a highly paid lawyer.

h. The Nobel Prize winner found the solution with a computer.

i. The sportsman hunted deer with a rifle.

j. The hoodlum broke the window with a stone.

This observation is familiar to those who do corpus-based studies of the semantics of particular words or construction types: many actually occurring instances do not easily fit into categories defined a priori, but the instances are semantically related and sometimes almost form a continuum, as in the examples in (3a-j). In this exemplar-based approach to semantics, meanings are mapped onto a very fine-grained conceptual space. As a result, the meaning of a word such as with is actually deeply embedded or intertwined with its context, both linguistic (as in the constructed examples in 3a-j) and nonlinguistic (in actual usage).

I now return to C's examples with the perspective of this exemplar-based, context-sensitive representation of the meaning of individual linguistic forms. Consider the examples of the simultaneity and adversity uses of while in the history of English cited by $\mathrm{C}$, from Hopper and Traugott (2003:91; I have added the final invented Modern English example from Hopper and Traugott):

(4) a. Jaet lastede pa [xix] winttre wile Stephne was king

'That lasted those 19 winters while Stephen was king' (ChronE [Plummer] 1137.36)

b. Whill others aime at greatnes boght with blod,

Not to bee great thou stryves, bot to bee god

'While others aim at greatness that is bought with blood, you strive to be not great but good' (1617, Sir W. Mure, Misc. Poems xxi.23)

c. While you like peaches, I like nectarines.

Simultaneity and adversity appear to be very different meanings. But as $\mathrm{C}$ notes, following Hopper and Traugott, events reported in discourse as simultaneous are often in contrast as well, and this has led to the extension of the meaning of while from simultaneity to contrastivity. $\mathrm{C}$ interprets this as a metonymic extension, because simultaneity and adversity are associated in a particular utterance such as (4b). But it can be looked at in a different way. Each of the utterances in (4a-c) represents a whole situation type. Even if we focus solely on what the 
connective contributes to the situations expressed in (4a-c), we see that there is indeed conceptual similarity between (4a) and (4b), and between (4b) and (4c). Most crucial for the semantic map model, there is a lower degree of similarity between (4a) and (4c); this is crucial because what makes the semantic map model valuable is the ability to posit different degrees of similarity. The similarity is found in the real world, where simultaneity and adversity occur together in the construal of paired situations for communicative purposes. But it is similarity nonetheless.

The example of English while is just one data point. A typological study would reveal just how close these situation types really are. I would predict with some confidence that a typological study would show that the semantic relationship between clauses in (4a) and (4c) is less close than that between either of those situation types and the situation type in (4b). These three types would not exhaust the conceptual space. Hopper and Traugott (2003) for example mention that in German, weil has become causal (and only very recently has it become concessive, via the causal path according to Hopper and Traugott). But my point here is that one must take a more holistic view of the situation types being represented in a conceptual space, and then the metonymic examples may turn out to represent a kind of similarity after all.

Even the lexical semantic examples I mentioned above may be amenable to a more holistic approach. 'Moon' and 'month' seem very different semantically. But in the frame-semantic or semantic domain approach to meaning introduced by cognitive linguistics (see especially Fillmore 1982, 1986; Langacker 1987, ch. 4; a survey is found in Croft and Cruse 2004), 'moon' and 'month' are in the same semantic frame, or more precisely, the same complex matrix (Langacker) of frames/domains which includes the celestial object itself and its periodic appearance and movement in the sky over time. I have argued that metonymy involves a shift in which a domain in a matrix (or a facet of a word's meaning, to use Cruse's terminology) is highlighted and in which a concept in the frame is profiled (Croft 1993/2002). The semantic domain matrices for 'moon' and 'month' are therefore very similar, differing only in which domain or facet is highlighted, and therefore also which concept is profiled. This is only part of the story. We do not have an explanation for which concepts are profiled when a different domain is highlighted. We also do not have an explanation for why some metonymies are more common than others (e.g. 'sun' is far less likely to be extended to 'day' than 'moon' is to 'month'). But a major part of the relationship is similarity in a holistic sense: both concepts share highly similar domain matrices.

This is not to say that all cases of different meanings subsumed under the same form can or should be interpreted as similarity. Frame-semantic similarity constitutes a shared semantics of a different type than similarity of concepts that are paradigmatic alternatives, or the intermediate case of holistic, contextually-defined similarity that is found in invited inference in grammaticalization. Nor is it to say that similarity is all that is going on in semantic change. But similarity - conceptual similarity as represented in the minds of speakers-does play a major role in typological patterns, synchronic and diachronic.

\section{References}

Brown, Cecil H. 1979. A theory of lexical change (with examples from folk biology, human anatomical partonomy and other domains). Anthropological Linguistics 21.257-76.

Croft, William. 1993/2002. The role of domains in the interpretation of metaphors and metonymies. Cognitive Linguistics 4.335-70. Revised version printed 2002 in Metaphor and 
metonymy in comparison and contrast, ed. by René Dirven and Ralf Pörings, 161-205. Berlin: Mouton de Gruyter.

-----. 2000. Explaining language change: An evolutionary approach. Harlow, Essex: Longman.

-----. 2001. Radical construction grammar: Syntactic theory in typological perspective. Oxford: Oxford University Press.

-----. 2003. Typology and universals, 2nd edition. Cambridge: Cambridge University Press.

-----. to appear. Exemplar semantics. To appear in a volume edited by Seana Coulson. Stanford: Center for the Study of Language and Information. Available online at http://www.unm.edu/ wcroft/.

Croft, William, Clayton Beckner, Logan Sutton, Jon Wilkins, Tanmoy Bhattacharya and Daniel Hruschka. 2009. Quantifying semantic shift for reconstructing language families. Poster, 83rd Annual Meeting of the Linguistic Society of America, San Francisco, California, January.

Croft, William and D. Alan Cruse. 2004. Cognitive linguistics. Cambridge: Cambridge University Press.

Croft, William and Keith T. Poole. 2008. Inferring universals from grammatical variation: Multidimensional scaling for typological analysis. Theoretical Linguistics 34.1-37.

Dahl, Östen. 1985. Tense and Aspect Systems. Oxford: Blackwell.

Evans, Nicholas. 1992. Multiple semiotic systems, hyperpolysemy, and the reconstruction of semantic change in Australian languages. Diachrony within synchrony: Language history and cognition, ed. by Günter Kellermann and Michael D. Morrissey, 475-508. Frankfurt: Lang.

Fillmore, Charles J. 1982. Frame semantics. Linguistics in the morning calm, ed. by The Linguistic Society of Korea, 111-137. Seoul: Hanshin.

----. 1985. Frames and the semantics of understanding. Quaderni di semantica 6.222-254.

Heine, Bernd, Ulrike Claudi and Friederike Hünnemeyer. 1991. Grammaticalization: A conceptual framework. Chicago: University of Chicago Press.

Hopper, Paul and Elizabeth Traugott. 2003. Grammaticalization, 2nd edition. Cambridge: Cambridge University Press.

Langacker, Ronald W. 1987. Foundations of Cognitive Grammar, vol. 1: Theoretical prerequisites. Stanford: Stanford University Press.

Levinson, Stephen, Sérgio Meira and the Language and Cognition Group. 2003. 'Natural concepts' in the spatial topological domain: Adpositional meanings in crosslinguistic perspective: An exercise in semantic typology. Language 79.485-516.

Schlesinger, Itzchak M. 1979. Cognitive structures and semantic deep structures: The case of the instrumental. Journal of Linguistics 15.307-24. 
Author's contact information:

William Croft

Department of Linguistics

Humanities Bldg. 526

University of New Mexico

Albuquerque, NM 87131-1196

USA

wcroft@unm.edu 